\title{
Mucinous Adenocarcinoma of the Colon Mimicking an Abdominal Wall Cellulitis
}

\author{
Kaczynski ${ }^{* 1}$, Wilczynska $\mathrm{M}^{2}$, Jaber $\mathrm{B}^{1}$ and Hilton $\mathrm{J}^{1}$ \\ ${ }^{1}$ ABM University Health Board, General Surgery Department, Morriston Hospital, Swansea, United Kingdom \\ ${ }^{2}$ University Hospital Llandough, Repiratory Medicine Department, Llandough, United Kingdom
}

*Corresponding author: Kaczynski J, ABM University Health Board, General Surgery Department, Morriston Hospital, Swansea, SA6 6NL, United Kingdom, Fax: 00441792703584, Tel: 00441792702222, E-mail: Jakub. Kaczynski@hotmail.co.uk

Citation: Kaczynski J, Wilczynska M, Jaber B, Hilton J (2014) Mucinous Adenocarcinoma of the Colon Mimicking an Abdominal Wall Cellulitis. J Case Rep Stud 2(4): 404. doi: 10.15744/2348-9820.2.104

Received Date: February 15, 2014 Accepted Date: March 03, 2014 Published Date: August 08, 2014

\section{Introduction}

Cutaneous metastases from abdominal malignancies are rare and have been reported in less than 5\% of patients [1]. Furthermore, metastases in patients suffering from colorectal neoplasia are even rarer entity. Tan et al, among 2538 of the new cases of colorectal cancer over the period of 6 years, reported only 3 cases $(0.1 \%)$ with cutaneous deposits [1]. Presentation varies from cutaneous or subcutaneous small nodules, rash or large fungating lesions [1-4]. Inevitably, their presence implies the disease progression, and poor prognosis with the reported survival between 1 to 34 months $[2,3,5]$.

We report a case of an elderly patient who initially was thought to present with a simple skin infection. Subsequently, the patient was diagnosed with the moderately differentiated mucinous adenocarcinoma and required the right hemicolectomy. This case highlights that a high index of suspicion is recommended in an unresolving skin erythema.

\section{Case Report}

An 89 year old female was referred by a general practitioner with 4 weeks history of an anterior abdominal wall cellulitis and an abscess discharging pus. She has received three courses of oral antibiotics with no improvement. The clinical history was obtained from the carers, family members and general practitioner as the patient was unable to provide any information due to dementia. There was no history of abdominal pain, change in bowel habit, weight loss or dysuria. Past medical history included diverticular disease, gallstones, hiatus hernia, hypothyroidism, atrial fibrillation, abdominal aortic aneurysm, right sided Spigelian hernia and dementia. The patient was a non-smoker with no alcohol consumption. She lived alone but was fully dependant for all the activities of daily living.

On admission, the patient was afebrile with normal observations. Examination of the cardiovascular and respiratory systems was unremarkable. On palpation, the abdomen was soft and mildly tender in the left iliac fossa with no evidence of an abscess. The right sided Spigelian hernia was incarcerated but non tender. Additionally, there appeared to be a sinus located suprapubically, which was surrounded by cellulitis. On probing, the sinus was continuous with a tract, which was discharging purulent material suggesting an underlying fistula. Digital rectal examination was normal. Laboratory tests showed normal white cell count of $5.9 \times 109 / 1$ and an elevated C reactive protein of $115 \mathrm{mg} / \mathrm{l}$. The remaining biochemical markers such liver function tests, amylase and electrolytes were normal. The urine dip stick was negative.

The patient was started on intravenous antibiotics (Cefuroxime and Metronidazole) and intravenous fluids. The provisional diagnosis of a colo-cutaneous fistula due to diverticulitis was made. Subsequently, a computed tomography (CT) of the abdomen/ pelvis was requested in order to exclude malignancy and plan surgical management accordingly. CT revealed inflammatory changes in the lower abdominal wall (Figure 1) and raised the suspicion of a fistula involving the right Spigelian hernia (Figure 2). This was confirmed by a fistulogram, which demonstrated contrast in the caecum (Figure 3). For that reason, the right hemicolectomy was performed. The operative findings revealed that the right Spigelian hernia contained the terminal ileum, which has fistulated through the anterior abdominal wall. The reminder of the laparotomy was unremarkable. The operation was uneventful, and the patient has made a good post operative recovery and was discharged. 


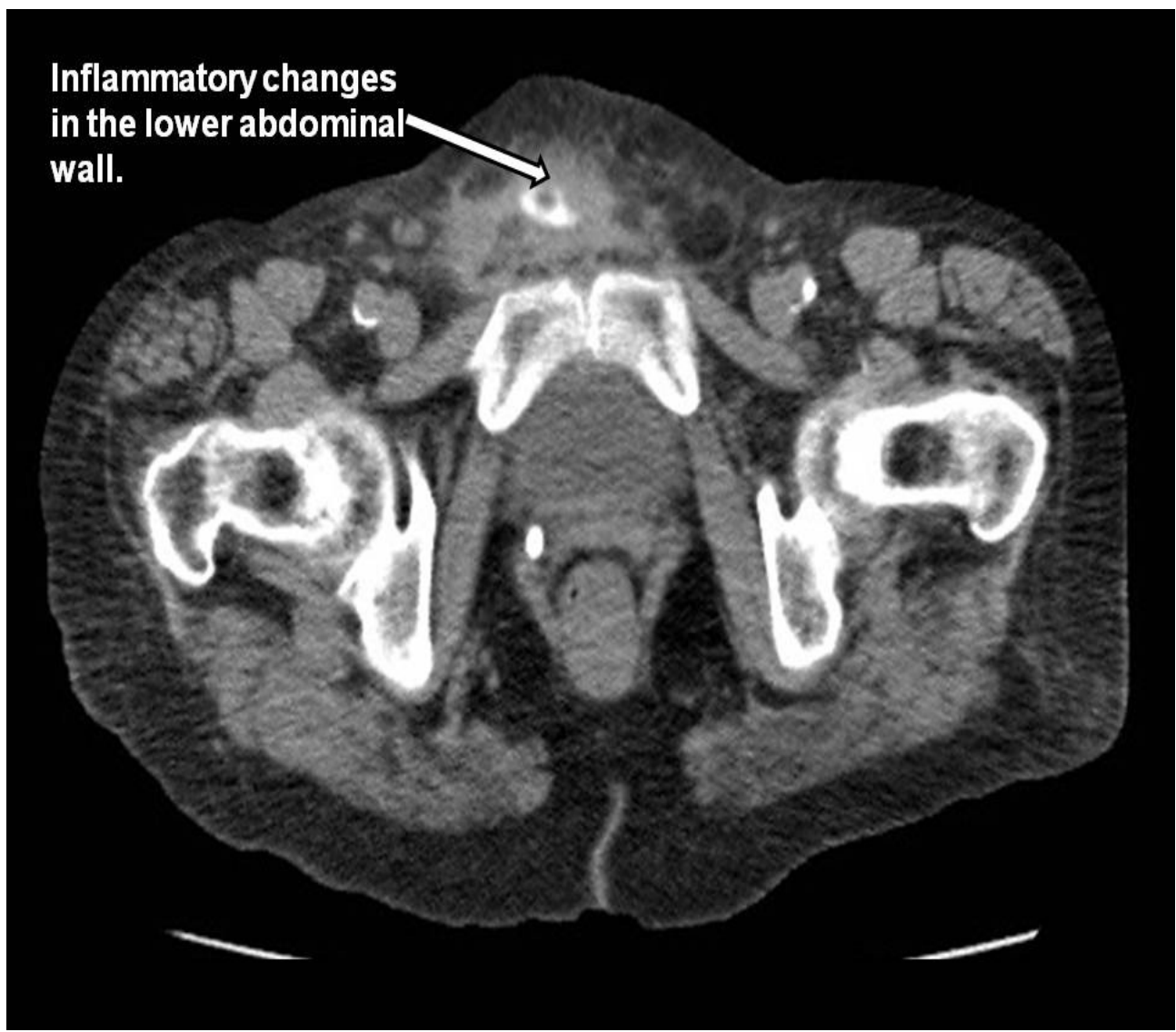

Figure 1: Inflammatory changes in the lower abdominal wall.

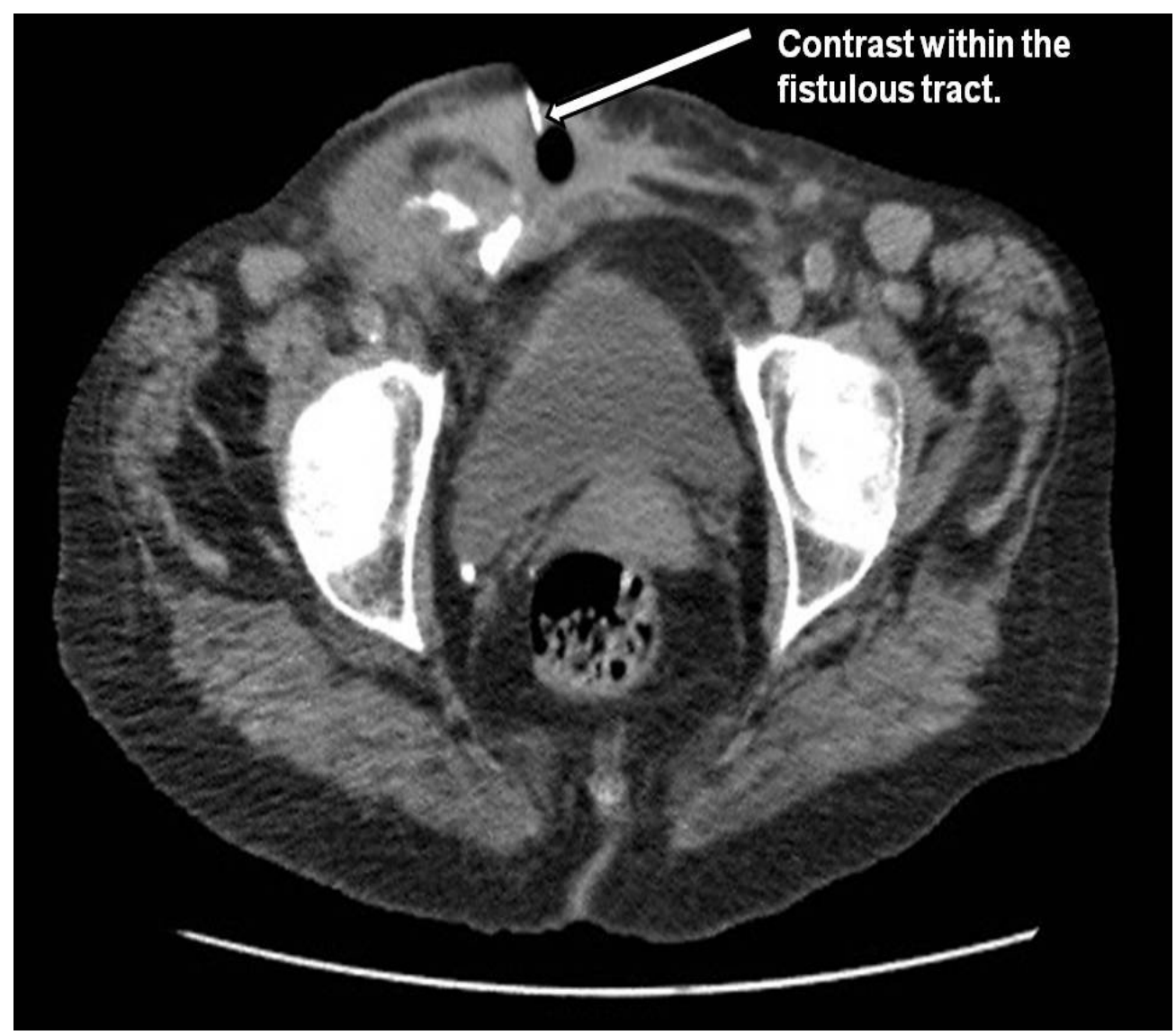

Figure 2: Fistula arising from the right Spigelian hernia. 


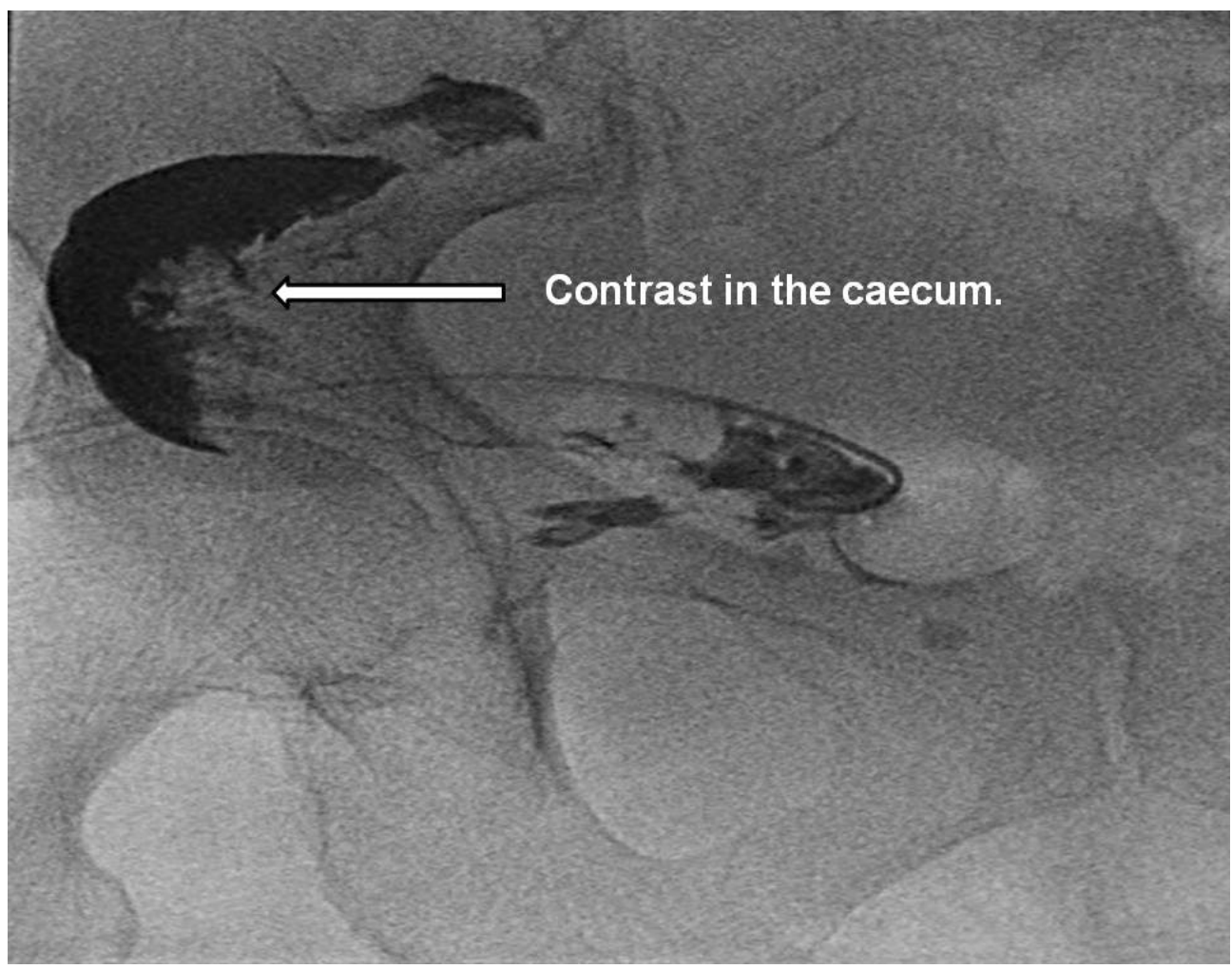

Figure 3: Fistulogram, demonstrating the terminal ileum fistula with contrast in the caecum.

Interestingly, the histopathology report showed moderately differentiated mucinous adenocarcinoma of the colon with no extra mucosal vascular invasion (Figure 4). These findings were discussed at the multidisciplinary colorectal meeting, and it was decided that no further treatment was required. However, 6 months later the patient was readmitted with a large fungating abdominal wall mass (Figure 5). CT chest/abdomen/pelvis revealed large necrotic mass $(75 \mathrm{~cm} \mathrm{x} 8 \mathrm{~cm})$ in the anterior lower abdominal/pelvic area associated with the omental deposits, necrotic intra-abdominal and inguinal nodal masses (Figure 6). The patient refused any form of intervention. The possibility of the surgical excision or radiotherapy has been discussed in detail with the patient's family members, who felt that neither of the treatment options were appropriate giving the worsening dementia and general deterioration of the patient's condition. The patient was discharged with a follow-up, which she did not attend.

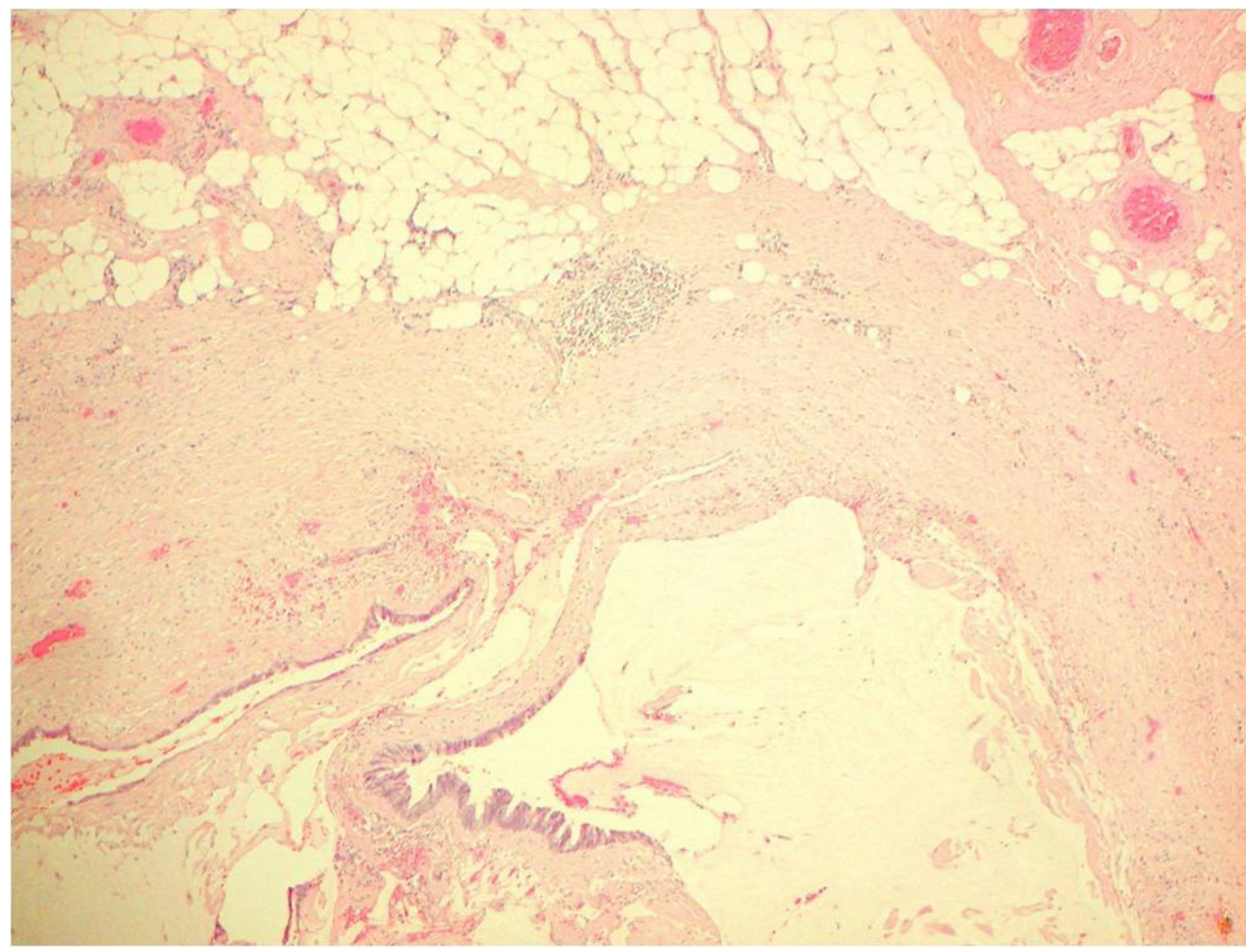

Figure 4: Mucinous adenocarcinoma infiltrating subcutaneous fibrofatty tissue. 


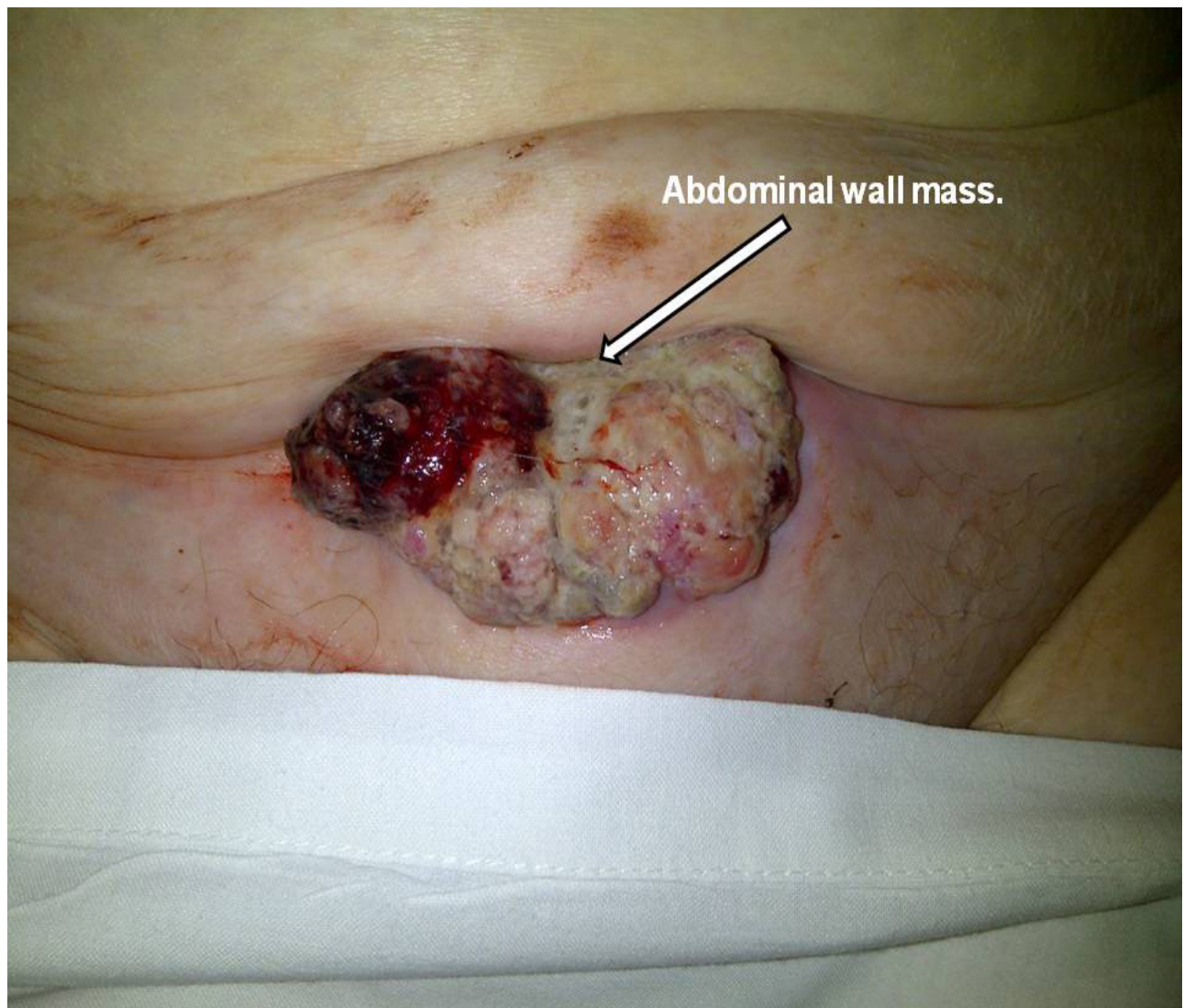

Figure 5: Large fungating abdominal wall mass.

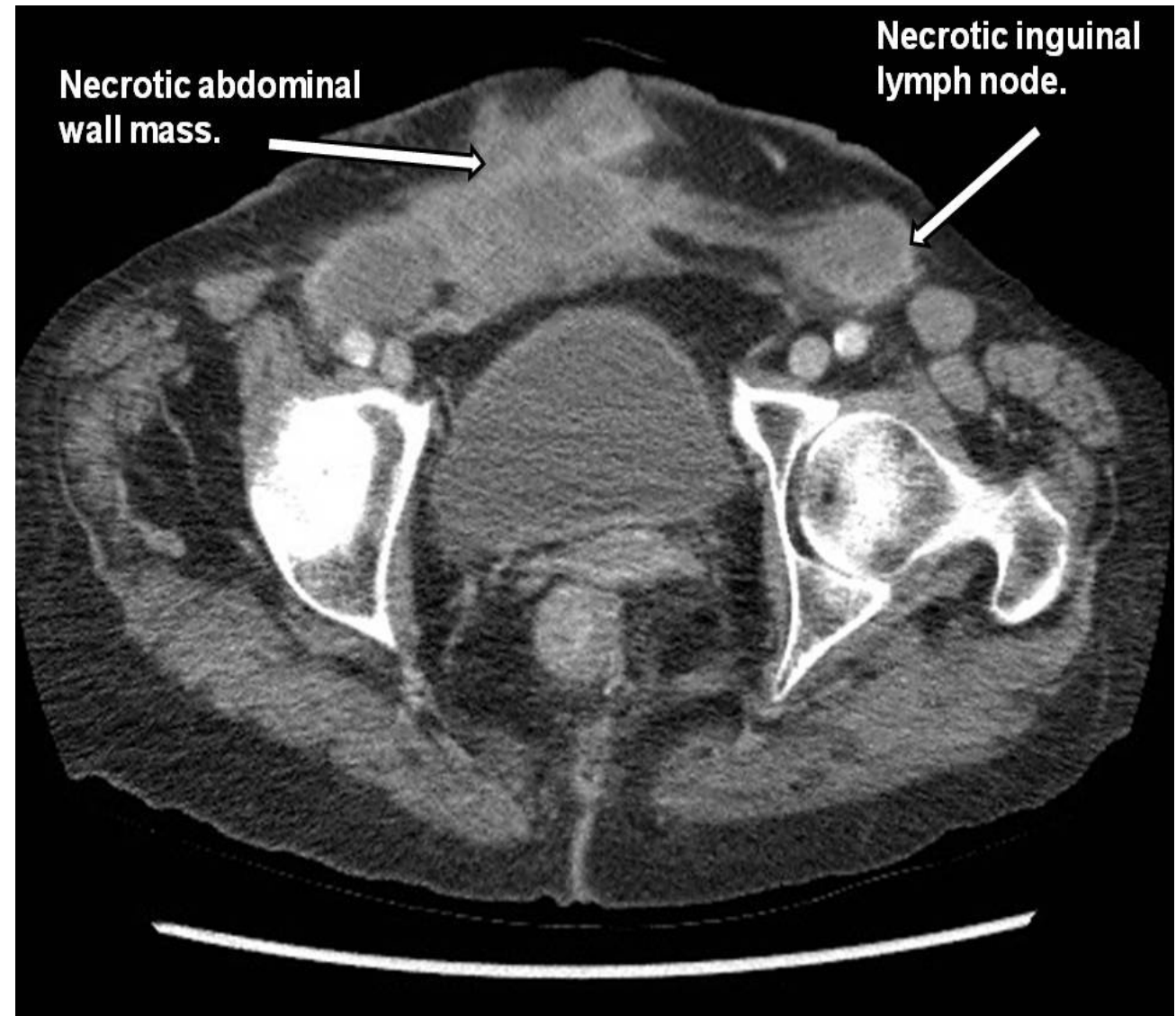

Figure 6: Large necrotic mass in the anterior lower abdominal/pelvic area with necrotic inguinal nodal mass 


\section{Discussion}

The commonest site for cutaneous metastasis in colorectal cancer is the abdominal wall at previous surgical scars including laparoscopic ports sites and colostomies $[2,6,7]$. This seems to support the direct model of the metastatic spread such as transmural tumour extension or implantation during surgery (iatrogenic) [2,3]. On the other hand, deposits at the remote locations from the original surgery sites have been reported. These include cases with facial, neck, scapular, anterior chest wall, back and lower limbs lesions [1,2,6-8]. This implies lymphatic or haematogenous spread of colorectal adenocarcinoma [2].

The presence of a skin lesion in the majority of cases indicates cutaneous metastases. However, further investigations are essential in order to rule out the possibility of a metachronous tumour, which can occur from 4 up to 30 years following an original resection [2]. Therefore, the first line investigation should be the biopsy of a skin lesion followed by the full body CT to assess for the metastases elsewhere [1,2]. The recommended treatment option of an isolated cutaneous deposit is a wide surgical excision followed by an adjuvant chemotherapy [2]. Whereas, conservative approach with radiotherapy are advised for non resectable skin lesions [2]. However, there are no clear guidelines regarding the treatment options, which reflect the small number of clinical cases encountered in the clinical practice.

\section{Conclusion}

In the described case, although the patient declined both the biopsy and surgical treatment, the clinical presentation favours the skin metastasis rather than the metachronous tumour. Nevertheless, this case highlights that perforating colonic cancer can present as a fistula, which can be easily mistaken for a common bacterial skin infection. Clinicians must remember that skin metastases are inflammatory in nature and often can present with an induration and well demarcated erythema [1]. This is thought to be due to a retrograde tumour invasion, which causes capillary congestion and vascular obstruction [1,7]. Therefore, any unexplained or persistent cellulitis type picture not responding to antibiotics therapy should be investigated further.

\section{References}

1. Tan K, Ho KS, Lai J, Lim JF, Ooi BS, et al. (2006) Cutaneous and subcutaneous metastases of adenocarcinoma of the colon and rectum. Ann Acad Med Singapore 358: 585-7.

2. Vijayasekar C, Noormohamed S, Cheetham MJ (2008) Late recurrence of large peri-stomal metastasis following abdomino-perineal resection of rectal cancer. World J Surg Oncol 6: 96.

3. Takami M, Hanada M, Kimura M, et al. (1983) Adenocarcinoma arising at a colostomy site. Dis Colon Rectum 261: 50-2.

4. Lookingbill DP, Spangler N, Helm KF (1993) Cutaneous metastases in patients with metastatic carcinoma: a retrospective study of 4020 patients. J Am Acad Dermatol 292: 228-36

5. Kauffman CL, Sina B (1997) Metastatic inflammatory carcinoma of the rectum: tumor spread by three routes. Am J Dermatopathol 195: 528-32.

6. Stavrianos S, McLean N, Kelly C, et al. (2000) Cutaneous metastasis to the head and neck from colonic carcinoma. Eur J Surg Oncol 265: 518-19.

7. Adani G, Marcello D, Anania G, , Mazzetti J, Jorizzo EF, et al. (2001) Subcutaneous right leg metastasis from rectal adenocarcinoma without visceral involvement. Chir Ital 533: 405-7.

8. Stanisławski M, Gruszecki L, Pawlak J (2008) Case of A 46-Year-Old Woman With Cecum Adenocarcinoma Metastasis to The Right Pectoralis Major Muscle and Skin. Pol Przegl Chir 803: 153-56.

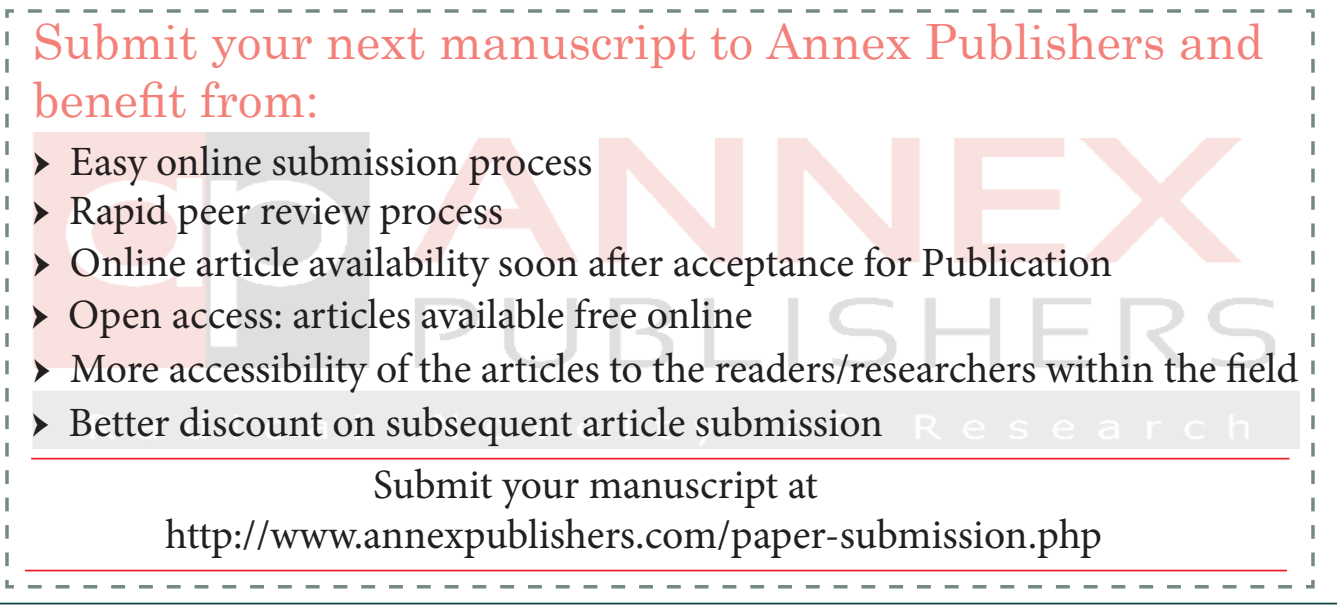

